\title{
Mindanao/Südphilippinen: Hintergründe eines vergessenen Krieges
}

\author{
Von Rainer Werning
}

Uber 70000 Tote, ungezählte Verletzte und weit mehr als eine halbe Million Flüchtlinge bzw. Vertriebene - das ist die vorläufige Bilanz eines Krieges, der zu Beginn der 70er Jahre im Süden des fernöstlichen Inselstaates offen ausbrach.

Schenkt man der Marcos-Regierung Glauben, so sind dessen Ursprünge denkbar einfach; It. Manilas Interpretation nämlich reduziert sich der Konflikt schlicht auf religiöse Zwistigkeiten zwischen Moslems und Christen.

So eingängig eine solche Lesart klingen mag, so weit ist sie davon entfernt, den komplexen Ursachen des Konflikts nachzuspüren.

Vier zentrale Aspekte gilt es im Auge zu behalten, welche - sich historisch teilweise überlappend - die Entstehung der Widerstandsbewegung in Gestalt der Moro National Liberation Front (MNLF) zu Beginn der 70er Jahre begünstigten:

(1) War es der Bevölkerung in den Südphilippinen während der spanischen Kolonialzeit (von ca. 1570 bis 1898) weitgehend gelungen, aufgrund einer relativ fortschrittlichen Sozialstruktur mit ausgeprägter Arbeitsteilung die Unabhängigkeit zu wahren, so setzte (2) mit Beginn der US-Herrschaft (1898-1946) ein Prozeß direkter Kolonialisierung und Kolonisierung ein, wodurch der vormals wirtschaftspolitisch blühende Süden von seinen regionalen Handelspartnern abgeschnitten und sukzessiv peripherisiert wurde. (3) Die in dieser Zeit unter der Ägide Washingtons genährte und protegierte (christliche nord)philippinische Elite (vorwiegend Großgrundbesitzer) übernahm nach der Unabhängigkeit i. J. 1946 vis-à-vis dem Süden die Funktion einer zentralen Ordnungsmacht, deren Wirtschaftspolitik auf Exportorientierung und Weltmarktintegration abzielte und zwangsläufig die Einbindung des "rückständigen Südens" in die Republik der Philippinen forcierte. Sichtbarster Ausdruck dieses Prozesses war die Verhängung des Kriegsrechts im Herbst 1972, durch das es jedweden politischen Protest gegen diese Strategie auszuschalten galt. (4) Dies wiederum markierte folgenschwere Konsequenzen innerhalb der Widerstandsbewegung selbst, deren Entstehung und Entwicklung im Vordergrund des nachfolgenden Beitrags stehen.

\section{Beginn des organisierten Widerstands}

Am 1. Mai 1968 unterzeichnete der frühere Gouverneur von Cotabato (der "Reiskammer Mindanaos"), Datu Udtog Matalam, ein Manifest in Pagalungan (Cotabato), das 
die Errichtung eines islamischen Staates - der Republik von Mindanao und Sulu - vorsah, in dem alle muslimischen Regionen der südlichen Philippinen vereint sein sollten. Dies signalisierte den Beginn der auf Sezession drängenden Muslim Independence Movement, die später in Mindanao Independence Movement (MIM) umbenannt wurde. Am 4. Juli 1969 sollte der neue Staat offiziell aus der Taufe gehoben werden. ${ }^{1}$

Durch solche Ereignisse alarmiert, begannen christliche Großgrundbesitzer, vielfach Holzindustrielle, mit der Rekrutierung von Mitgliedern diverser Bürgerwehrtruppen zum Zweck des Aufbaus von Privatarmeen und bewaffneten Banden, die wenig später unter der Bezeichnung Ilagas (Ratten) unter der Zivilbevölkerung Furcht und Schrecken säen sollten und von der Constabulary und den regulären Streitkräften bei zunehmender Eskalation der Gewalt gedeckt wurden. Ende 1975/Anfang 1976 sollten sie gut 35000 Mann stark gewesen und vielfach von Bürgermeistern und Provinzgouverneuren angeführt worden sein.

Massaker im März, Juni, Juli und August 1971, denen Moslems durch Terroranschläge von Ilagas gegen Moscheen und Barrios zum Opfer fielen, führten unter den Moslems nunmehr ihrerseits zur Aufstellung bewaff neter Trupps, die in Cotabato als Blackshirts und in Lanao als Barracudas firmierten. Bis September/Oktober 1971 sind in beiden Lanao-Provinzen, Cotabato und Bukidnon mindestens 100000 Menschen zu Flüchtlingen geworden. Seit dieser Zeit wandten die philippinischen Streitkräfte in größerem Stil "search and destroy"-Operationen an, was Libyen, Malaysia und Kuwait auf internationaler Ebene erstmals von einem "beginnenden Völkermord" sprechen ließen. Operationen der Streitkräfte hielten unvermindert an und lösten einen Aufschrei der Entrüstung aus, als Militärs nach den Regional- und teils nachgezogenen Senatswahlen im November 1971 auf einen Trupp gerade von den Wahlurnen zurückgekehrter, unbewaffneter Moslems im Barrio Tacub (Kauswagon, Provinz Lanao del Norte) das Feuer eröffneten und über 40 Menschen töteten. Angestrengte Verfahren gegen die dafür als verantwortlich angeklagten 21 Soldaten, darunter 3 Offiziere, sind im Laufe der Zeit versandet. Manila war nun seinerseits bemüht, im Ausland eine diplomatische Offensive einzuleiten und lud zu Beginn des Jahres 1972 zwei Delegationen mit Beobachtern aus Ägypten, Indonesien, Malaysia, Singapur, Pakistan, Iran, Irak, Saudi-Arabien und (später dann auch aus) Libyen ein, um den Vorwurf des Genozids entkräftet zu wissen. Die Delegatio-

1 Diese und die weiteren Ausführungen basieren auf Interviews, die der Autor im Sommer 1977 in den Lanao-Provinzen und im Sommer 1978 auf Jolo, Siasi und Tawi-Tawi (Sulu-Archipel) mit MNLF-Kadern führte, wie auf folgenden Quellen: verschiedenen Ausgaben von "Mahardika", dem of fiziellen MNLF-Bulletin; des "Far Eastern Economic Review" (Hongkong); von "Liberation", dem of fiziellen Organ der Nationaldemokratischen Front (NDF); von "Ang Bayan", dem zentralen Organ der Kommunistischen Partei der Philippinen (CPP), sowie auf: Robert D. McAmis, "Muslim Filipinos: 1970-72«: in: "Solidarity", 8.6:3-16, Manila 1973, Solidaridad Publishing House; Peter G. Gowing, "Constrasting Agenda for Peace in the Muslim South" in: "Philippine Quarterly of Culture and Society," 8.4:286-302, Cebu City, Dec. 1980, University of San Carlos; Alunan C. Glang, "Muslim Secession or" Integration?, Quezon City 1969, R. P. Garcia Publishing Co.; Mamintal A. Tamano, "Report on Deteriorating Peace and Order Conditions in Cotabato," filed by the Committee on National Minorities, Senate of the Philippines, Manila (April) 1971 und "An Anatomy of Philippine Muslim Affairs," Makati/Rizal 1971, Filipinas Foundation, Inc. 
nen schlossen sich zwar in offiziellen Verlautbarungen dem Vorwurf einer "staatlich gelenkten Völkermordkampagne" nicht an, wiesen aber nachdrücklich auf die Gefahr verstärkter, teilweise religiös motivierter kriegerischer Auseinandersetzungen hin.

Genau einen Monat nach Verhängung des Kriegsrechts, am 21./22. Oktober 1972, stürmten 500-1000 vorwiegend jugendliche Maranaos aus 7 verschiedenen Untergrundorganisationen Marawi City, drangen auf das Gelände der "Mindanao State University" vor, bemächtigten sich der dort installierten Radiostation, über die sie als "Revolutionsrat für die Unabhängigkeit Mindanaos" (MRCI) die Beseitigung der Regierung, die sie als Nachfolgerin des spanischen und amerikanischen Regimes und als Instrument zur Unterdrückung des Islams werteten, propagierten. Sie attackierten ein nahegelegenes Hauptquartier der Constabulary, plünderten Läden christlicher Geschäftsleute und töteten einige der von ihnen zuvor als Geiseln genommenen Christen.

\section{a. MIM und $M N L F$}

Führend in der MIM waren außer Datu Udtog Matalam der frühere Kongreßabgeordnete Raschid Lucman wie der einstige Senator aus Lanao del Sur, Domocao Alonto. Innerhalb der Moro-Gesellschaft zählten alle drei zur dünnen Elite und verfügten über weitreichende Beziehungen. Ihre ausgeprägt religiöse, mitunter fundamentalistisch gewendete Argumentation, derer sie sich in ihrer Kritik gegen die Regierung in Manila bedienten, ermöglichte ihnen beträchtliche finanzielle Zuwendungen aus dem In- und Ausland (Naher und Mittlerer Osten). Die genaue Höhe dieser Finanzhilfen ist unbekannt. Die sezessionistische Ausrichtung der MIM, also ihre angestrebte vollständige Loslösung Mindanaos und der anderen südlichen Inseln aus dem philippinischen Staatsverband, muß unter zwei Aspekten gesehen werden: (1) war es nicht länger mehr möglich, angesichts der Durchdringung der Region durch die vereinte Gewalt multinationaler Konzerne und des ihren Interessen dienenden Marcos-Regimes in politischer Abstinenz zu verharren, was zumindest der MIM-Führung die politische Legitimation entzogen hätte, und (2) sollte ihr Insistieren auf staatliche Eigenständigkeit einer politischen Avantgarderolle entsprechen bei gleichzeitiger Umgehung/Ausklammerung einschneidender sozialpolitischer und wirtschaftlicher Zielvorstellungen, zu deren Opf er sie schließlich selbst werden könnte. Mit anderen Worten: die muslimische Elite ward, wollte sie sich des Rückhalts in der Bevölkerung vergewissern, in die Rolle eines nach außen sich antikolonial, antiimperialistisch gebenden Kämpfers gedrängt, dem jedoch schon aus materiellen Erwägungen daran gelegen sein mußte, antikoloniale, antiimperialistische Inhalte zu verschleiern bzw. sie durch die Propagierung eines noch zu schaffenden Staates darinselbst aufgehoben zu wissen.

Berichte, wonach Malaysia und Indonesien die MIM mittels finanzieller Hilfe zum politischen Durchbruch ihres Ziels zu verhelfen gedachten, sind nicht eindeutig nachweisbar. Diesem Ansinnen dürften angesichts der innerhalb der seit 1967 bestehenden 
ASEAN* strikt auf Vermeidung jeglicher staatlichen Grenzverschiebung und/oder sezessionistischer Ambitionen bedachten Politik kaum reale Erfolgschancen einzuräumen gewesen sein, zumal seit der Niederlage der USA in Indochina und dem unrühmlichen Ableben der SEATO (Südostasiatischen Paktorganisation) die übergeordnete Staatsraison in den jeweiligen Mitgliedstaaten der ASEAN es gebot, winterne Unruheherde" und ein Uberschwappen wie immer auch gearteter zentrifugaler Tendenzen im Keim zu ersticken. Die "Befriedung" Osttimors und das Einfrieren des seit den 60er Jahren zwischen Kuala Lumpur und Manila schwelenden Grenzproblems (der von Manila geltend gemachte Anspruch auf den ostmalaysischen Staat Sabah) bekräftigen dies aufs anschaulichste.

Auf Initiative der MIM-Führung sollen muslimische Jugendliche zum militärischen Training nach Malaysia, ins Grenzgebiet zu Thailand, entsandt und dort von Palästinensern und britischen Söldnern im Dschungelkampf ausgebildet worden sein. Die Zahl dieser ersten Gruppe wurde auf 90 Mann veranschlagt - 67 Maranaos, 8 Maguindanaos und 15 Tausugs/Samals. Ein Mitglied dieser Gruppe war Nur Misuari, einst Politologie-Lehrer an der "University of the Philippines" und später führender Organisator der Moro National Liberation Front (MNLF). Misuari soll diese Zeit im Ausbildungslager gemeinsam mit Freunden (zusammen bildeten sie 1969 den Nukleus der später offiziell vollzogenen Gründung der MNLF) genutzt haben, um politische und wirtschaftliche Probleme Mindanaos zu diskutieren, was innerhalb der MIM weitgehend unterblieben war.

Nach einem mehr als einjährigen Auslandsaufenthalt kam die Gruppe 1971 nach Mindanao zurück. Bald darauf erfolgte in Zamboanga City ein Treffen der politisch fortschrittlichsten und aktivsten Mitglieder der Gruppe, auf dem die historischen Erfahrungen des Moro-Kampfes und die Rolle der MIM analysiert wurden. Dabei wurde letzterer vorgeworfen, keine überzeugende Perspektive zu haben und über kein klares Programm zu verfügen. Die MIM, so ein weiterer Kritikpunkt, sei aufgrund ihrer Kollaborationsbereitschaft mit Marcos außerstande, die Unabhängigkeit der Moros zu garantieren. Nach eingehenden Diskussionen über Formen des bewaffneten und parlamentarischen Kampfes wurde die MNLF formal gegründet und Misuari zum Vorsitzenden ihres Zentralkomitees gewählt.

Die MNLF begann mit einer gezielten Rekrutierungskampagne unter muslimischen Jugendlichen. 1972 gingen 300 Jugendliche aus dem Cotabato-Gebiet nach Malaysia und unterzogen sich dort einer militärischen Ausbildung. In Lanao wurden, nachdem dort die als militärischer Arm der MIM konzipierten "Blackshirts" entstanden waren, die Shock Troops und die Revolutionary Army gegründet, um sich gegen Ubergriffe seitens Großgrundbesitzer und paramilitärischer Constab ulary-Einheiten (PC) zu verteidigen. Die gesamte Lanao-Region zählte mit Cotabato zu den ersten Gebieten, wo die PC spätestens seit 1970 "search and destroy"-Kampagnen durchführte, was Regierungs-

* Vereinigung südostasiatischer Nationen, zu deren Mitgliedstaaten neben den Philippinen Indonesien, Singapur, Malaysia und Thailand gehören. Anfang Januar '84 schloß sich ihr der Olstaat Brunei an. 
stellen (z. B. die "Social Welfare Administration") im September 1971 bereits von 50000 Flüchtlingen allein in Lanao del Norte sprechen ließ. Außerdem entstand unter den Moros die IKHLAS-Bewegung (Jugendliche für den Islam), die von religiösen Führern, ulamas, befehligt wurde, und maßgeblich an dem bereits erwähnten "MarawiAufstand" Ende 1972 beteiligt war.

Die Rekrutierungskampagne muß zumindest in militärischer Hinsicht spektakuläre Erfolge gezeitigt haben; schon wenige Monate nach Verhängung des Kriegsrechts kam es überall im Süden zu großen bewaff neten Auseinandersetzungen zwischen Regierungstruppen und MNLF-Verbänden bzw. deren militärischer Arm in Form der Bangsa Moro Army, deren Stärke Mitte der 70er Jahre gut 20000 Mann betragen haben dürfte. Im März 1973 griffen Moro-Guerilleros in 27 Bezirken Cotabatos Kontingente der philippinischen Streitkräfte (AFP) an, sprengten Brücken, bemächtigten sich strategischer Verbindungswege, stürmten Militärlager und überrannten etliche militärische Kontrollposten. In nur wenigen Wochen verloren über 1000 AFP-Soldaten ihr Leben. Diese hohen Verluste in der Frühphase des Mindanao-Krieges erklärten sich aus der Tatsache, daß auf den Schultern junger Rekruten und aus dem Norden und den Visayas abkommandierter, kampfunerfahrener Leutnante die Hauptlast eines Kampfes ruhte, dessen Ursprünge/Gründe, Terrain und Milieu ihnen völlig fremd bzw. feindselig gesonnen waren.

\section{b. Eskalation der Gewalt}

Kampfbomber und -hubschrauber wurden nebst aufgestockten Truppenkontingenten verstärkt im Süden eingesetzt. Kriegsschiffe kreuzten in allen größeren Häfen auf und begannen mit der gezielten Bombardierung bestimmter Küstenstreifen. In dieser Zeit wurden erstmalig Napalmeinsätze gegen "verdächtigte Rebellengebiete" von Mactan und Zamboanga City aus geflogen. Ob US-Piloten einen Teil dieser Einsätze selbst flogen, ist mit höchster Wahrscheinlichkeit anzunehmen. Als gesichert indes gilt, daß amerikanische Instrukteure von der Clark Air Base philippinische Bomberpiloten u. a. auch in die "Technik" des Napalmkrieges unterwiesen.

Mitte 1973 bildete die Region Zamboanga den Hauptschauplatz des Krieges, der sich zum Jahreswechsel 1973/1974 zunehmend auf Tawi-Tawi und Jolo verlagerte. Waren Ortschaften wie Parang, Bilaan und Maimbung bereits von Moro-Einheiten gestürmt worden, so erfolgte am 7. Februar 1974 der Angriff auf die Hauptstadt Jolo. Nach der Bombardierung von Militärinstallationen der Regierungstruppen bemächtigten sich die Moros des Flughafens, des Hafengebiets und gesamten Stadtkerns, der "Notre Dame University" sowie des Militärkomplexes "Camp Asturias". Die Kommunikationslinien zwischen den verschiedenen AFP-Einheiten in Sulu und dem Hauptquartier in Zamboanga City waren zeitweilig unterbrochen.

Bald wurden von Zamboanga City aus Bombergeschwader und Kriegsschiffe gen Jolo in Bewegung gesetzt. Hatte die MNLF zunächst versucht, die Regierungstruppen in die umliegenden Berge zu locken, so setzte sie jetzt auf einen Entscheidungskampf zweier 
Armeen in der Stadt. Aus politischen Erwägungen traute die MNLF den Regierungstruppen nicht zu, gegen die Zivilbevölkerung dieser stolzesten aller muslimischen Städte im Süden mit offenem Terror vorzugehen. AFP-Verbände schossen die Stadt aus der Luft und von der See buchstäblich in Schutt und Asche. In nur einer Nacht gab es 2000 Tote und 60000 Flüchtlinge!

Hatte die MNLF (nicht zuletzt durch die im Ausland genossenen Trainingsmethoden) bis zur Zerstörung Jolos eine militärische Taktik konventioneller Kriegsführung und statischer Defensive verfolgt, so wurde sie seit Februar 1974 gezwungen, Aspekte der Mobilität, der Úberraschungsattacken in den Vordergrund zu rücken, um die eigenen Kräfte zu schonen und die soziale Massenbasis zu vergrößern. Diese Taktik zielte auf die Zermürbung/Aufweichung denn auf einen Entscheidungssieg in offener Feldschlacht ab. Die AFP beantworteten dies ihrerseits mit einer Úberführung massiver militärischer Unterdrückung in eine Politik verstärkter Okkupation. Parallel mit einer ca. 700\%igen Aufstockung der Militärausgaben von 1972-76, dem Anschwellen der Ist-Stärke der AFP von reichlich 60000 auf 250000 Mann im gleichen Zeitraum und einem wachsenden militärischen Engagement der USA wurde das Entstehen von Banden wie den Civilian Home Defense Forces (CHDF) und pseudoreligiöser Sekten gefördert, deren Mitglieder einige Zehntausende stellen. Der Einsatz von Napalm, Splitterbomben und Giftgasen und die nicht nur der Terminologie, sondern auch dem Charakter nach dem Modell des Vietnamkrieges folgenden Operationen und Programme - wie "base denial", "resource control", "rural development", "salvaging", "search and destroy", "strategic hamlets", "zona" - bildeten die Klammern, um die Bevölkerung in Botmäßigkeit zu halten. Aus dem Okkupationscharakter der AFP machten führende Militärs wie Marcos und sein Verteidigungsminister Enrile keinen Hehl. "Wer immer die Fähigkeit der AFP testen möchte", so erklärte Juan Ponce Enrile damals, "mag dies tun. Sollte Blutvergießen notwendig sein, so muß halt Blut fließen." ${ }^{2}$

Außer der Eingliederung von Polizeieinheiten in die Armee fand innerhalb derselben eine Zentralisierung der Kommandostruktur statt, welche Manila seit 1976 im Kampf gegen die MNLF als notwendig erachtete.

Am 1. Mai 1976 wurde Mindanao unter ein vereintes Militärkommando, das Südkommando, gestellt, an dessen Spitze Admiral Romulo Espaldon trat, ${ }^{3}$ der heute das Amt eines "Ministers für muslimische Angelegenheiten" bekleidet. Der damalige AFP-

2 Zit. nach: "Moving Heaven and Earth: An Account of Filipinos Struggling to Change Their Lives and Society", ed. by the Commission on the Churches' Participation in Development (CCPD), World Council of Churches and the Philippine Ecumenical Writing Group, Manila 1982, p. 183.

3 Integriert in dieses Südkommando wurden folgende Einheiten: 1) das "Südwestkommando" mit Sitz in Zamboanga City; 2) das in Cotabato ansässige "Zentralmindanao-Kommando"; 3) die in Davao stationierte Einsatztruppe "Pagkakaisa" ("Einheit"); 4) die "4. Infanteriebrigade" in Cagayan de Oro City unter dem Befehl von Brigadegeneral Alfonso Alcoseba; 5) sämtliche zur "4. Constabulary" zählenden Einheiten; 6) das "Sulu-See- und Grenzkommando“; 7) die von Brigadegeneral Abraham Mangonon, Zamboanga, bef ehligte "1. Luftlandedivision" und 8) die "Südflotte" der Marine unter Kapitän Pablo Gutierrez. 
Generalstabschef, General Romeo C. Espino, begründete die Zusammenfassung der ehemaligen Teilverbände in ein integriertes Kommando mit der Dringlichkeit, die Truppenmobilität zu erhöhen und die sich als hinderlich erwiesenen Kompetenzschwierigkeiten in Einsatzfällen auszumerzen. ${ }^{4}$

Vorab war die Bereitstellung eines 50 Mio. US-Dollar umfassenden militärischen Beschaffungskredits erfolgt. "Ich möchte", so äußerte sich Marcos, "keinen Bef ehlshaber sehen, der mir mit dem Argument in den Ohren liegt, er habe soundsoviele Mann verloren, nur weil Waffen und Gerät veraltet seien." Nach der Zerstörung Jolos wurde die Flottenkapazität der Kriegsmarine um $60 \%$ erhöht. Flugzeuge vom Typ "Phantom F4A" wurden durch neuere "F4E"-Mehrzweckkampfbomber, die obsoleten "F86 Sabre-" durch "Skyhawk A4"-Maschinen ersetzt und das Arsenal britischer "Scorpion"-Panzer beträchtlich aufgestockt. ${ }^{5}$ Ergänzt wurde dieses Anschaffungs- und Modernisierungsprogramm durch eine Heraufsetzung der US-Militärhilfe um $64 \%$, ${ }^{6}$ wobei Sonderzuweisungen für die verschiedenen Geheimdienste des Landes noch unberücksichtigt blieben.

Trotz all dieser Maßnahmen konnte der MNLF nicht das Rückgrat gebrochen werden. Unter der muslimischen Bevölkerung genoß die Organisation wachsende Sympathie und stellte einen zumindest militärisch ernstzunehmenden Faktor dar. Ausschlaggebend für das Úberleben der MNLF waren ihre den Erfordernissen eines langwährenden Zermürbungskrieges angepaßte militärische Taktik einerseits und die Expansion der Neuen Volksarmee (NPA) auf der anderen Seite.

War es den AFP Mitte der 70er Jahre gelungen, strategisch bedeutsame Verbindungslinien der NPA im Nordosten (Provinz Isabela) und Süden (Provinz Quezon) Luzons zu durchschneiden, so vermochte die NPA dort schon bald ihre Kräfte im Westen, in der Bicol-Region wie im zentralen Hochland neu zu gruppieren und auf mehreren Inseln der Visayas Fuß zu fassen. Um einen Krieg an zwei oder gar mehreren Fronten zu vermeiden, setzte Manila 1975/76, freilich nicht ungeschickt, auf die Karte politischer Annäherung durch Verhandlung, was angesichts wachsender Proteste im In- (dort vor allem seitens bürgerlicher Oppositioneller und erstmals größerer Teile der Kirchen) und Ausland immer dringlicher wurde. Für die MNLF sollten Marcos' Verhandlungspolitik, die ihr zugrundeliegenden konkreten Schritte und schließlich die Reaktion im Lager ausländischer Befürworter zu einer schweren Belastungsprobe werden.

4 Seit dem 1. Mai 1976 kann der Befehlshaber des Südkommandos, ohne die Zustimmung der betreffenden Kommandeure der Teilstreitkräfte einzuholen, Armee-, Luftwaffen-, Constabulary- und Marineverbände an die Front werfen. Siehe "Bulletin Today" (Manila), May 2, 1976, pp. 1,5 - Vgl. auch: R. Werning, "Südostasien im Jahre des Drachen: Politischer Reisebericht", Bonn 1976, S. 9-39.

5 "Pacific Stars and Stripes", Dec. 22, 1975.

6 Von 58,433 Mio. US-Dollar für die Fiskaljahre 1970-72 auf 95,862 Mio. US-Dollar für den Zeitraum 1973-75. 


\section{Vom Schlachtf eld zum Verhandlungstisch}

Die 6. Islamische Außenministerkonferenz, welche vom 12.-15. Juli 1975 in der saudiarabischen Stadt Dschidda tagte, verabschiedete eine Resolution, in der die Regierung in Manila zu Konsultationen mit der MNLF auf der Basis eines vom ministeriellen "Komitees der Vier" entworfenen Arbeitspapiers unter Aufsicht des Islamischen Sekretariats auf gefordert wurde. Dieses Komitee, gebildet von den Außenministern (bzw. ihren Repräsentanten) Libyens, Saudi-Arabiens, des Senegal und Somalias, hatte einen Text vorgelegt, dessen Kernpunkt die Gewährleistung einer Selbstregierung der Inseln Mindanao, Basilan, Sulu und Palawan nim Rahmen der nationalen Souveränität der Philippinischen Republik" bildete. Während die Diplomaten um geschliffene Textformulierungen bemüht waren, bekundete Manila nach kurzweiligem Zögern sein allgemeines Einverständnis mit diesem Vorschlag und nutzte die Zeit, da die MNLF durch die Befolgung der Dschidda-Empfehlung in ihren Aktionen zeitweilig paralysiert war, zur Straffung seiner militärischen Verbände auf Mindanao. Munitionsdepots konnten ungestört angelegt bzw. aufgefüllt, die Feuerkraft somit erhöht und eine Reorganisierung der Kommandostruktur fernab größerer Gefechte geplant werden.

Marcos' Haltung gegenüber der MNLF erfuhr keinerlei Bruch in ihrer Kontinuität. Eine verschlagene Zuckerbrot-und-Peitsche-Politik, Bombenterror und Bestechung, signalisierte Konsultationsbereitschaft und das Unterlaufen einmal getroffener Vereinbarungen erlebten unter seiner Ägide eine bemerkenswerte Synthese. Gewiß ist, daß jene Zeit der Konsultationen Manila weitaus größere Erfolge bescherte als der MNLF, aus deren Reihen denn auch etliche Kader und Mitglieder deprimiert ausscherten, sei es aus Verbitterung über die zeitweilige Einstellung des Kampfes, was einmal mehr ihre mehr militaristische denn politische Linie unterstrich, oder aus der Erwartung materieller Pfründe, die man sich durch ein Arrangement mit Marcos versprach. So verließ, um nur eins von vielen Beispielen zu nennen, der ehemalige Rechtsanwalt Abdul Hamid Lucman die Reihen der MNLF, wurde von Marcos mit Schmiergeldern reichlich bedacht und reiste 1975 als Berater zu dem MNLF-Verhandlungsteam nach Dschidda. Während eines am 8. März 1976 geführten Gesprächs erklärte Admiral Romulo Espaldon, Kommandeur des wenig später geschaffenen Südkommandos, daß Hadji Hamid Camlian, ehemals Präsident der im Frühjahr 1974 ausgerufenen Bangsa Moro Republic (Bangsamoro Republik), der sich Ende 1975 in Manila ergab, nunmehr Gouverneur von Basilan sei.

Mit reichlichem Propagandapomp wurde eine Amnestie für Mitglieder der MNLF/BMA verkündet. Und es vergingen kaum Tage, an denen nicht irgendwelche "Rebellen aus dem Süden“ im Malacañang-Palast zur zeremoniellen Waffenübergabe aufkreuzten. Der überwiegende Teil dieser "Deserteure" war indes niemals in der MNLF/BMA aktiv. Vielfach handelte es sich schlicht um Banditen oder herumstreunende Abenteurer, die gewinnbringend auf der "Amnestiewelle" zu reiten vermochten. In der Berichterstattung sollte so die Nutzlosigkeit jedweden regimekritischen Protests 
unterstrichen, die angeblich wohlwollende, auf Ausgleich und Versöhnung bedachte Politik der Regierung hervorgekehrt und die MNLF/BMA diskreditiert werden. Anfang 1976 erklärte Marcos, daß seine Regierung 133 Mio. US-Dollar für ein Moslem-Rehabilitierungsprogramm bereitgestellt habe.? Davon allerdings seien, so meldete das "Minsupala-Bulletin" im Januar 1976, Unsummen an Schmiergeldern prominenten Deserteuren der MNLF/BMA zugeschoben worden. ${ }^{8}$

Während eines Rotary Club-Treffens unterstrich der damalige in Manila akkreditierte US-Botschafter, William H. Sullivan, anläßlich der Zusammenkunft zwischen Ford und Marcos Ende 1975, daß Washington die "sezessionistischen Tendenzen im Süden" als Bedrohung amerikanischer Sicherheitsinteressen in Südostasien betrachte. ${ }^{9}$ Als ehemaliger Botschafter in Laos $^{10}$ und letzter Botschafter seines Landes unter dem persischen Schah Reza Pahlevi-Regime war Sullivan wegen seiner engen Beziehungen zur CIA bekannt und aufgrund langjähriger Antiguerillaerfahrung nicht ohne Hintergedanken auf den Botschafterposten in Manila berufen worden.

\section{a. Die Phase der Konsultationen - das Abkommen von Tripolis}

Bis Mitte 1974 propagierte die MNLF die Loslösung des Südens aus dem philippinischen Staatsverband und die Errichtung einer unabhängigen islamischen Republik. Die Resolution Nr. 18 der 5. Islamischen Außenministerkonferenz ${ }^{11}$ in Kuala Lumpur (21.-25. Juni 1974), die Gesprächsrunden zwischen Vertretern der MNLF und der Marcos-Regierung im Januar 1975 im saudiarabischen Dschidda, die bereits erwähnte 6. Islamische Außenministerkonferenz im Sommer desselben Jahres und weitere gemeinsame Konsultationen in Libyen im November 1976 bildeten die entscheidenden Etappen auf dem Wege des schließlich am 23. Dezember 1976 in der libyschen Hauptstadt Tripolis unterzeichneten Abkommens zwischen Manila und der MNLF. Im Vordergrund all dieser Gesprächsrunden wie im Tripolis-Abkommen selbst standen 1) die politische und friedliche Lösung des Konflikts im Süden wie 2) Autonomie für die Moros "innerhalb der nationalen Souveränität und territorialen Integrität der Philippinischen Republik", folglich eine von der MNLF selbst vollzogene Rücknahme ihrer maximalistischen Forderung nach staatlicher Eigenständigkeit. ${ }^{12}$

7 Zit. nach: "South China Morning Post" (Hongkong), March 2, 1976.

8 Minsupala ist die Abkürzung für Mindanao, Sulu und Palawan.

9 "Pacific Stars and Stripes", Dec. 13, 1975.

10 Sullivan wardort u. a. an der Aufrechterhaltung des von der CIA unterhaltenen "Air America"-Flugnetzes und der Ausbildung der Söldnertruppe des berüchtigten Generals Vang Pao beteiligt.

11 Der Text dieser Resolution ist abgedruckt in: "The Southwestern Philippines Question", 2nd ed., Manila 1977: Department of Foreign Affairs, R.P., (Annex IV, pp. 40-50.)

12 Als Dokumente zur Einschätzung des "Tripolis-Abkommens" und der Ereignisse danach, die den Standpunkt aller Beteiligten widerspiegeln, seien folgende genannt: 1) Der eigentliche Text ist in vollem Wortlaut im "The Southwestern Philippines Question", op. cit., Annex XI, pp. 76-82, abgedruckt; 2) die Regierungsposition findet sich im "White Paper on Ceasefire and Terrorist Atrocities in Southwestern Mindanao and Sulu", Manila: Department of Public Information, Oct. 29, 1977; 3) Marcos' Version über das Scheitern der 
Im Abkommen wurden folgende wesentlichen Punkte aufgelistet:

a) Die autonome Region der Moslems im Süden umfaßt 13 Provinzen - Basilan, TawiTawi, Sulu, Zamboanga del Sur, Zamboanga del Norte, North Cotabato, South Cotabato, Maguindanao, Sultan Kudarat, Lanao del Norte, Lanao del Sur, Davao del Sur und Palawan nebst allen dort gelegenen Städten und Dörfern;

b) die Außenpolitik fällt in den Zuständigkeitsbereich der Zentralregierung in Manila;

c) im autonomen Gebiet haben die Moslems das Recht, ihre eigene Gerichtsbarkeit (Sharia-Gesetze) auszuüben. Sie sollen außerdem in allen Gerichten, den Obersten Gerichtshof inbegriffen, vertreten sein;

d) den Moslems wird das Recht eingeräumt, in ihren Gebieten Schulen und Universitäten zu errichten;

e) im autonomen Gebiet sollen eine Legislativversammlung und ein Exekutivrat auf dem Wege direkter Wahlen entstehen;

f) nicht später als am 20. Januar 1977 soll ein Waffenstillstandsabkommen in Kraft treten;

g) durch eine vollständige Amnestie, durch die Freilassung politischer Gefangener wie durch die Rückführung der Flüchtlinge soll die Bewegungs- und Versammlungsfreiheit garantiert werden;

h) eine spezielle regionale Friedenssicherungstruppe soll innerhalb der autonomen Region geschaffen und

i) die Errichtung einer provisorischen Regierung vorgesehen werden, um die Wahl der Mitglieder der Legislativversammlung einzuleiten.

Details des Tripolis-Abkommens sollten auf bilateralem Wege in der 1. Hälfte des Jahres 1977 ausgehandelt werden, was Manila außerordentlich entgegenkam.

Kaum war das Abkommen unterzeichnet, beschuldigte Manila die MNLF, AFPTruppen aus dem Hinterhalt attackiert zu haben, während die MNLF der Regierung vorwarf, "unseriös, verräterisch und scheinheilig" zu handeln und das Abkommen bewußt zu unterlaufen. Nur Misuari richtete eine bittere Kritik an die Adresse Manilas, indem er Marcos beschuldigte, auf die Teilung der autonomen Regierung in zwei Exekutivräte mit einem Regionalparlament hinzuarbeiten, wodurch der im Abkommen vorgesehene einheitliche Charakter der autonomen Regierung gesprengt würde. Ferner weigere sich Manila, so Misuari, seine bewaffneten Streitkräfte abzuziehen, bewaffnete Banden aufzulösen und Platz für den Aufbau einer speziellen Friedenssicherungstruppe,

Tripolis-Nachfolgegespräche, damit auch des Abkommens, war in seiner am 3. Mai 1977 vor der "InterimBatasang Pambansa" (Nationalversammlung) gehaltenen Rede enthalten, deren Wortlaut wiedergegeben ist in: A. T. Tiamson/R. N. Cañeda (comp.), "The Southern Philippine Issue: Readings in the Mindanao Problem", Vol. 1, Marawi City 1979: 12th Annual Seminar on Mindanao and Sulu Cultures, Nov. 16-18, 1979, pp. 1-18; 4) Abdurasad Asani, Direktor des MNLF-Verbindungs- und Informationsbüros in Tripolis, legt in der Schrift "Moros - Not Filipinos", published by the Bangsamoro Research Centre, 1980, n.p., 43 pp. die offizielle MNLF-Position dar; Nur Misuari gab seine Einschätzung in seiner Rede auf dem "International Congress on Cultural Imperialism" im Oktober 1977 in Algier kund; 5) eine weitere Lageeinschätzung stammt von der gegen die MNLF opponierenden "Bangsa Moro Liberation Organization" (BMLO) in: "Position Paper", Mecca, Saudi Arabia: BMLO General Headquarters, August 1978, 15 pp. typescr. 
deren Kern aus BMA-Einheiten zu rekrutieren sei, zu machen. Seinen beiden letzten Kritikpunkten war besonderes Gewicht beizumessen; Manila beanspruchte nach wie vor die völlige Kontrolle sämtlicher Bodenschätze und Ressourcen in der anvisierten autonomen Region und führte ein Referendum-Plebiszit durch, das nirgends im Abkommen vorgesehen war. Dieses Referendum-Plebiszit wurde am 17. April 1977 abgehalten. Dabei ging es um die Frage, ob Mindanao eine oder zwei Verwaltungen erhalten und ob die MNLF die autonome Regierung allein oder gemeinsam mit anderen Kräften bilden sollte. Unter Kriegsrechtsbedingungen wäre es schon einer Sensation gleichgekommen, hätten sich die Wähler nicht mit einem "eindeutigen Nein" entschieden. Das Referendum-Plebiszit entbehrte nicht nur einer juristischen Basis; selbst in formaler und prozeduraler Hinsicht war es fragwürdig. Es wurde zu einem Zeitpunkt durchgeführt, da die provisorische Regierung nicht einmal bestand, Marcos 5 Schlüsselprovinzen kurzerhand als bereits halbautonom einstufte, welche keiner politischen Reorganisierung bedurften, und Manila erst noch im Prozeß der Schaffung des Vorbereitungskomitees für den Aufbau dieser provisorischen autonomen Regierung war. Des weiteren wurden Einladungen zur Teilnahme am Referendum-Pfebiszit bezeichnenderweise bestimmten Einzelpersonen der MNLF, nicht aber ihrer Führung zugeleitet. Was als Autonomie konzipiert war, entpuppte sich als grobschlächtiger Regierungsoktroi; 15 von 29 Sitzen der provisorischen Regierung waren für die MNLF reserviert, aus deren Reihen Manila aber selbst die ihm genehmen Vertreter ernannte. Die MNLF boykottierte - schon aus Gründen politischer Glaubwürdigkeit - das Referendum-Plebiszit und kehrte zu ihrem ursprünglichen Ziel der Sezession zurück.

\section{Die MNLF zwischen Anpassung und Widerstand}

Wie zu erwarten stand, entfalteten die zwischenzeitlich aufgerüsteten AFP- und paramilitärischen Truppen unter dem Vorwand des MNLF-Vertragsbruchs eine militärische Großoffensive (vor allem in Zamboanga del Norte und Basilan), in deren Verlauf es ihnen gelang, über die Ausdehnung ihres Operationsradius und Erweiterung ihrer Gebietskontrolle hinaus etliche der im Zuge vorangegangener Verhandlungsrunden bekannt gewordenen MNLF-Führer aufzuspüren, zu liquidieren oder durch Bestechungsmanöver auf die Regierungsseite herüberzuziehen. Letzteres traf nicht selten schon deshalb auf fruchtbaren Boden, weil schwankende MNLF-Kader in der Unterzeichnung des Tripolis-Abkommens einen modus vivendi mit Manila erblickten, der weitere Opposition hinfällig zu machen schien. Aus diesen Kräften rekrutierte sich denn auch die Führungsspitze in der von Manila eigenmächtig geschaffenen "Provisorischen Regierung der muslimischen Autonomen Zone" (MAZ). 


\section{a. Interne Widersprüche}

Zu keiner Zeit war die MNLF weiter von ihrem ursprünglichen Ziel eines souveränen Staates im Süden (der eventuell einmal das ostmalaysische Sabah einschließen sollte) entfernt, wie das im Jahre 1977 der Fall war. Die qua MNLF-Propaganda und -Rhetorik ausgegebenen Erfolgsmeldungen vermochten nagende innere Probleme nicht zu kaschieren, die durch außenpolitische Konstellationen noch vertieft wurden.

Zum einen erwies sich die Dezentralisierung der Kommandostruktur und das Aufbrechen großer Kontingente in kleine, mobile und regional operierende Einheiten als schwierig und wirkte sich nachteilig auf den gesamten Organisationszusammenhalt aus. Regionalistische Tendenzen und die für ein Kriegsherrentum instrumentalisierten persönlichen Loyalitätsbekundungen waren unverkennbar. Zudem hielten sich etliche ZKMitglieder, darunter auch Nur Misuari, in Libyen und anderen Nahoststaaten auf, so daß Direktiven nur durch Emissäre übermittelt werden konnten. Ethnische Rivalitäten, nie ganz verstummt, brachen unter solchen Bedingungen hervor und schürten Querelen zwischen Auslandsführung und lokalen Kommandeuren ebenso wie zwischen den vor Ort selbst agierenden Befehlshabern.

Eine politisch ausgewiesene Einheit innerhalb der MNLF-Führung blieb streckenweise gelinde ausgedrückt - zweifelhaft und der Praxis persönlicher Loyalitäten untergeordnet. Während Nur Misuari an der Vision eines souveränen Moro-Staates im wesentlichen festhielt, propagierte sein damaliger Stellvertreter und Angehöriger einer einflußreichen Grundbesitzerfamilie, Abul Khayr Alonto, unverblümt eine kompromißlerische Politik, ${ }^{13}$ was Marcos für seine Zwecke zu nutzen wußte.

Die finanzielle und diplomatische Unterstützung der MNLF erfolgte durch muslimische Länder, deren konservative Regimes in der Islamischen Konferenz (IC) stets das Ubergewicht stellten. Deren Untersuchungskommission ging niemals so weit, Marcos des Völkermords zu bezichtigen und offen die Propagierung eines eigenständigen MoroStaates auf ihre Fahnen zu schreiben. Die Marge der Entscheidungsfreiheit blieb für die MNLF nicht nur schmal, sondern ward durch die Empfehlungen der IC weitgehend eingeengt. Misuari machte daraus keinen Hehl, als er erklärte: "Aufgrund der Vermittlung der IC optierte die MNLF nur für eine 'vollständige politische Autonomie', anstatt ... die Befreiung der gesamten nationalen Heimat - Mindanao, Basilan, Sulu und Palawan - mit einer Gesamtfläche von $116895,3 \mathrm{~km}^{2}$ anzustreben. Von dem gesamten von uns beanspruchten Gebiet bleiben nurmehr ungefähr $60 \%$ der nationalen Heimat unseres Volkes übrig, beschränkt auf 13 Provinzen und 11 Städte. " ${ }^{14}$ Wenn dennoch die IC der MNLF trotz ihres Festhaltens am Konzept staatlicher Eigenständig-

13 Siehe: "Far Eastern Economic Review" (Hongkong), June 25, 1975.

14 Nur Misuari in seinem Redebeitrag auf dem "International Congress on Cultural Imperialism" in Algier im Okt. 1977. Zur Gesamtproblematik dieses Beitrages vgl. noch die Schrift "Die gestutzten Flügel der Freiheit - Menschenrechte und Militarisierung in den Philippinen", Münster 1985, die von mehreren philippinischen Menschen- und Bürgerrechtsorganisationen zusammengestellt wurde. 
keit nicht die Unterstützung versagt, so resultiert das aus der Einsicht, daß die MNLF unter den Moros die weitaus bedeutsamste Kraft darstellt und ein Entzug ihrer diplomatischen Unterstützung als Verrat am Prinzip islamischer Brüderlichkeit ausgelegt werden könnte. Daß die IC der MNLF-Maximalforderung wohl nur selbst geringe Aussicht auf Erfolg beimißt, ist an ihrem Verhältnis zu Manila ersichtlich, welches niemals den Punkt eines vollständigen Bruchs bzw. der Aufkündigung diplomatischer Beziehungen erreichte. $^{15}$

15 Vgl. zu diesem Beitrag die Karte auf S. 328. 


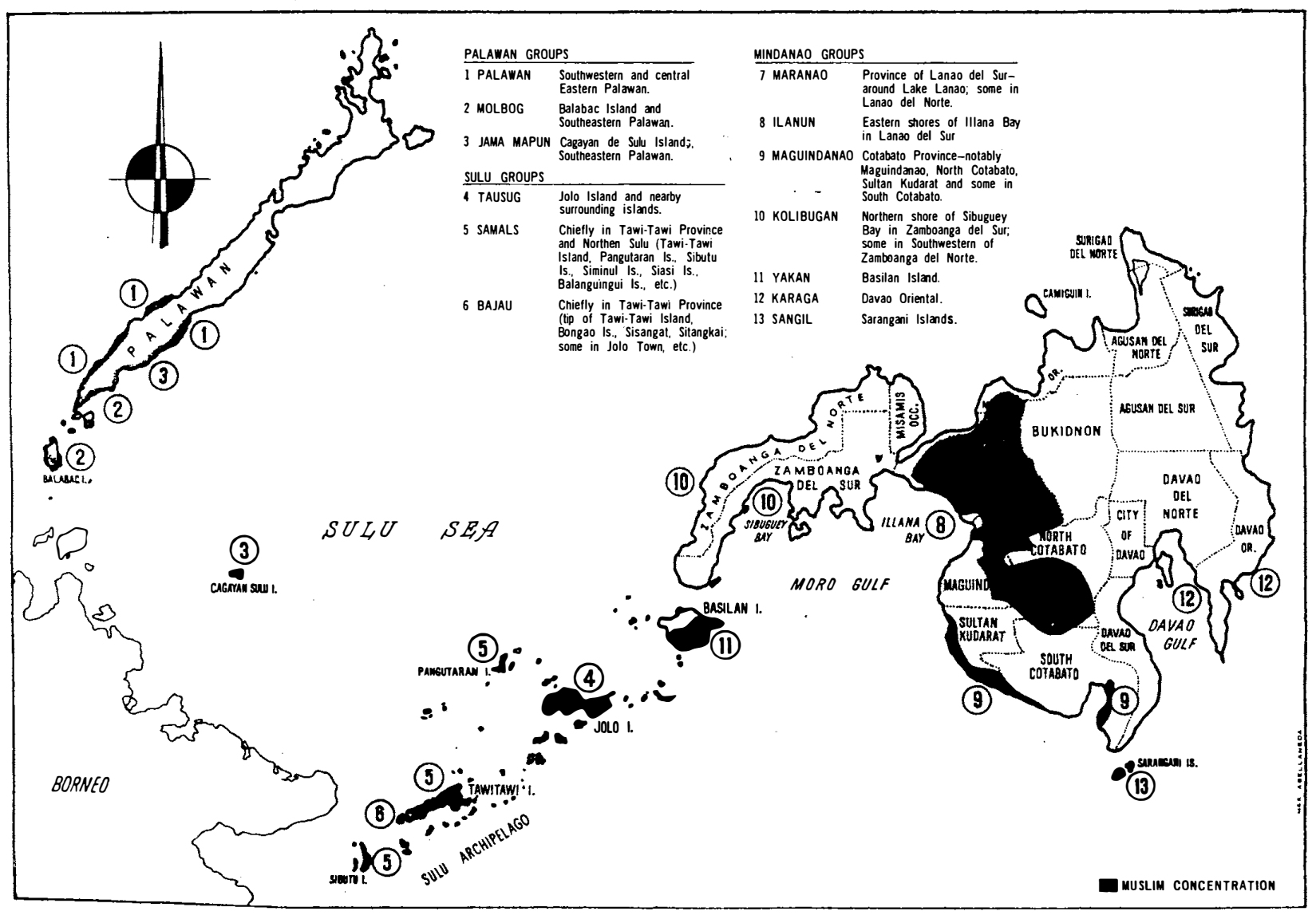




\section{'El grupo Monterrey، - zur sozio-ökonomischen Formation einer regionalen Machtgruppe Mexikos}

\section{Von Andreas von Schumann}

The rise of the "Grupo Monterrey" as a decisive force within the private sector has been a development parallel to the crisis of Mexico's political system which has been smouldering since the beginning of the seventies.

Although the industrialists of the north-eastern state of 'Nuevo Leon' have based their power on a diversified economy, their influence reaches beyond the economic sector. By initiating unions friendly to the employers and by organizing both company-run social security systems and private educational facilities, the "Grupo Monterrey" has successfully established its own mass support.

On the basis of economic and social ascendancy, this group confronts the postrevolutionary project of the "Partido Revolucionario Institucional" (PRI) with a socio-political alternative which has materialized out of a conglomeration of neo-liberal and socialchristian ideas.

The assessment of the socio-economic formation of this originally regional power group highlights the political impact of the "Grupo Monterrey" on determining Mexico's development.

\section{Mindanao/Südphilippinen: Hintergründe eines vergessenen Krieges}

\section{Von Rainer Werning}

Whereas more than three centuries of Spanish rule had failed to subdue the Muslim population - Moros - in the Philippine South, 48 years (1898-1946) of American administration set in motion the gradual subjugation of Mindanao and the Sulu archipelago. This was achieved through a four-pronged strategy, of direct military intervention (superior weaponry); internal colonisation (e.g. systematic transfer of surplus population from the North to the sparsely populated but resource-rich South); large-scale corporate investment and the creation/instrumentalisation of a basically Manila-centered (Christian) bureaucracy.

Since independence (1946) these processes have accelerated under the North-Filipino landed élite. Its shift in development strategy and emphasis on export-orientation and closer world market integration facilitated this group's iron-handed actions aimed at preserving its interests in close alliance with dominating foreign - largely North American - powers.

To uphold what was left of political self-determination, cultural identity and religious beliefs, the Moros resorted to armed resistance. The formation of the Moro National 
Liberation Front, 'MNLF' (1971) led the Marcos régime to proclaim martial law in autumn 1972. The stage was set for an escalating war that so far has left well over 70,000 people dead. From its very start the Philippine state maintained that the "forgotten " war be conducted on religious grounds, for this allowed the government to present itself as an arbiter of disputes between two religious communities, thus concealing the politico-economic content of the conflict.

The author closely examines the roots and early developments of the MNLF. Taking into consideration the relevant data and sources of all participants and having interviewed several MNLF cadres in the late seventies he then concentrates on the organisation's intrinsic difficulties to define a clear-cut theoretical framework for and practical pursuit of self-determination.

Ironically, lingering ethnic, politico-economic and cultural differences among the Moros were even reflected in the MNLF leadership. It frequently shifted from autonomy to secession and vice versa.

Being more of a military organisation, a successive decentralisation of the MNLF's command structures - partially due to its Libyan connection - made it difficult to sustain its cohesion. Political unity within the leadership became precarious. Various MNLF cadres had persisted in their different ethnic and political allegiances, and splits within the Central Committee - e.g. between Nur Misuari and Abul Khayr Alonto, Chairman and Vice Chairman respectively - were brewing.

Marcos was well aware of these developments. Temporarily decreasing military pressure, he offered as bait a political settlement based on regional autonomy which in his view was likely to bring dissensions into the open. In this context, the Islamic Conference (IC) was granted an opportunity to exercise its moderating influence. It had distanced itself from the MNLF's maximalist aspirations to sovereignty and kept a studied silence regarding the MNLF claim that the Moros were faced with genocide. A compromise, in form of the Tripolis Agreement (Dec. 23, 1976) with its ceasefire provision, was short-lived. As he had anticipated, Marcos gained more from it than he could ever have achieved on the battlefield. Internal strife within the MNLF sharpened. The "radical" faction under Nur Misuari was not opposed to strike a tactical alliance with the growing New People's Army (NPA) in order to break through the chronic political isolation of the MNLF, thus contributing to a partial secularisation of the Muslim movement. This, on the other hand, substantially narrowed the "moderates'" room to manoeuver. They surrendered or otherwise slipped under Manila's wings, a move which brought the IC and Marcos closer to each other than ever before.

Above all, both are anxious not to let a "local conflict" spill over, potentially to endanger regional stability. Nor can and will they be accomodative to a far-reaching secularisation and finally radicalisation of the Moro struggle which, for "reasons of security", in the eyes of the Association of Southeast Asian Nations (ASEAN) and Washington are likewise not to be tolerated. 